\title{
A Garch Model Test of The Random Walk Hypothesis: Empirical Evidence from The Platinum Market
}

\author{
Knowledge Chinhamu \\ School of Mathematics, Statistics and Computer Science, University of KwaZulu-Natal, \\ Private Bag X54001, Durban 4000, South Africa. \\ Email: Chinhamu@ukzn.ac.za
}

\section{Delson Chikobvu}

Department of Mathematical Statistics and Actuarial Science, University of Free State, Box 339, Bloemfontein 9300, South Africa. Email: Chikobvu@ufs.ac.za

\section{Doi:10.5901/mjss.2014.v5n14p77}

\begin{abstract}
The paper investigates whether there are periods when platinum prices follow the random walk process (weak-form efficient) and periods when they deviate from the random walk theory (mean reversion). Monthly log returns of platinum prices are examined using the Augmented Dickey-Fuller test (ADF) and a GARCH model with time-varying properties. A GARCH model with time-varying properties is able to capture periods when the random walk theory may be true and periods when it may be false. This study confirms the existence of random walk for platinum prices over the period January 1970 to May 2012. From the year 1999 to the year 2010, the drift parameter is positive and statistically significant. Therefore, the platinum market is regarded as weak-form efficient.
\end{abstract}

Keywords: GARCH model; Market efficiency; Random-walk hypothesis; mean reversion; Time-varying parameters.

\section{Introduction}

In a weak-form efficient market (random walk hypothesis), all information contained in historical prices is instantaneously reflected in the current market prices. This effectively precludes the opportunity to detect abnormal returns through a trend trading approach. However, some investment strategies are used to exploit trend in prices. In financial economics, the random walk hypothesis deserves further empirical analysis. Absence of the random walk according to a random walk test indicates the existence of the intertemporal dependence at some chosen lag. A rejection of the random walk hypothesis implies mean reversion in asset prices.

There has been a growing interest in precious metal markets by agents that incorporate metals in production process such as many metallurgic companies and also in jewelry industry, where metals such as gold, platinum and silver are clearly dominant. These characteristics imply that there has been a strong demand in these markets. Most research done focused on the analysis of the gold market. The main interest has been the role of this precious metal as a hedge against inflation. Little has been done with regard to other precious metals (silver, platinum and palladium). Hiller et al. (2006) concluded that financial portfolios that contain precious metals perform significantly better than standard equity portfolios. They also found that precious metals exhibit some hedging capability during periods of abnormal volatility. The thrust of the study is to investigate whether platinum prices are mean reverting or follow a random walk process. Platinum presents an interesting case because it is one of the most sought after metals in the world due to its properties and uses. It is thus important to investigate whether platinum price predictions can be done with accuracy. Forecasting precious metal prices, including platinum future prices, remain one of the biggest challenges facing econometricians and statisticians. Some research concludes that commodity prices, including platinum, follow a random walk, implying that tomorrow's expected prices should be the same as today's value. If platinum prices follow a random walk, then prices would be very difficult, if not impossible to predict. It is imperative to revisit mean reversion and the random walk in the context of platinum, as this has serious implication on modeling and forecasting platinum prices. The research is motivated by the fact that precious metals market could represent an important option for investors in order to diversify their investment strategies and portfolios. Conventional tests of the random walk hypothesis, for example the Lo and 
MacKinlay (1988) variance tests, lead to inconclusive conclusions about stock price index following a random walk at a predetermined significance level. Although such tests can be applied to successive time periods, they cannot readily capture gradual changes in efficiency over successive observations. There is a need to revisit the the random walk theory in commodity prices using other tests.

This paper investigates whether platinum prices are mean reverting or follow a random walk process at all time periods. The Augmented Dickey-Fuller (ADF) tests and the GARCH model with time-varying properties approach are used to investigate mean reversion and random walk processes in platinum prices. Whilst the techniques adopted in this paper may be standard in empirical finance, the approach as presented, with time varying parameters has not been applied to platinum prices to the best of our knowledge. There are still gaps that need to be updated to provide the empirical evidence for the random walk in the platinum market. This paper is of significance to applied statisticians, econometricians and investors. Researchers interested in modeling platinum and investigating market efficiency will find this paper relevant. The results have serious implications on modeling and forecasting of platinum prices as well as investing in platinum.

The rest of this paper is organized as follows. Section 2 gives an overview of the random walk model and related literature. Section 3 describes the empirical methods used in this study. In section 4, the data and results are reported and discussed. Section 5 provides a conclusion.

\section{Related Literature}

Random walk behavior and market efficiency in commodities markets are mainly examined using variance tests, unit root tests such ase the Augmented Dickey-Fuller (ADF), the Philips-Peron (PP), the Kwiatkowski-Philips-Schmidt-Shin (KPSS). Unit root tests are used to determine if the series is differenced or trend non-stationary as a necessary condition for a random walk. According to Andersson (2007), the traditional unit root tests have a low statistical power. Andersson (2007) proposed using hedging errors in option prices as a measure of the most appropriate stochastic process. This economic test is used to differentiate mean reversion and a random walk in 280 different commodities. The author concluded that commodity prices are mean reverting.

Bessembinder et al. (1995) analysed the relation between metal (gold, platinum and silver) price levels and slope of the futures term structure defined by the difference between a long maturity future contract and its first nearby. Assuming that future prices are unbiased expectations (under the real probability measure) of future spot metal prices, an inverse relation between prices and the slope constitutes evidence that investors expect mean reversion in spot prices, as it implies lower expected future spot prices when prices rise. The authors concluded the existence of mean reversion of metal price over the period 1982-1991.

Nwala (2011) used the Langrage Multiplier (LM) unit root tests with one and two structural breaks to investigate the random walk hypothesis for 12 commodity prices namely gold, silver, platinum, steel, and copper, uranium, aluminum, iron ore, lead, nickel, tin, and zinc. The data consisted of monthly observations spanning the time period from January 1986 through to December 2010. The preliminary results indicated that the commodity prices are unit root processes. The results implied that the community prices are characterized by a random walk.

\section{Methodology}

In this section the models used to investigate the random walk are discussed.

\subsection{A simple model for log returns}

We define the natural logarithmic return (simply log return) of platinum at time $t$ as:

$r_{t}=\log \left(P_{t} / P_{t-1}\right)=\log \left(P_{t}\right)-\log \left(P_{t-1}\right)=p_{t}-p_{t-1}$

where $\mathrm{P}_{\mathrm{t}}$ is the price of platinum at time $\mathrm{t}$.

The simplest model which can be used to test for the random walk is the simple auto-regressive (AR (1)) model, namely:

1. $r_{t}=\beta_{0}+\beta_{1} r_{t-1}+\varepsilon_{t}$

where $r_{t}=p_{t}-p_{t-1}$, is the log return of platinum price, $\beta_{0}$ and $\beta_{1}$ are the parameters that need to be estimated and $\varepsilon_{\mathrm{t}} \sim \operatorname{IID}\left(0, \sigma^{2}\right), \mathrm{p}_{\mathrm{t}}=\log \left(\mathrm{P}_{\mathrm{t}}\right)$ is the natural logarithm of the price of platinum at time $t$. If the platinum price follows a random walk, $\beta_{1}=0$ and so 
2. $p_{t}=\beta_{0}+p_{t-1}+\varepsilon_{t}$

which is a random walk with drift parameter $\beta_{0}$.

The natural logarithmic transformation reduces the impact of heteroskedasticity that may be present in large data sets with high frequency. The transformation also ensures that predicted platinum price is positive when anti-logs are taken. The model, however, does not cater for changing volatility.

Three versions of the random walk model are distinguished by Cambell et al. (1997) cited in Jefferis and Smith (2005) which depend on the assumptions of the error term, namely $\varepsilon_{\mathrm{t}}$. Under the first model, the error terms are independently and identically distributed with a zero mean and constant variance, denoted by $\varepsilon_{\mathrm{t}} \sim \operatorname{IID}\left(0, \sigma^{2}\right)$. In the second model, the error terms are independent but not identically distributed, which allows for unconditional heteroscedasticity in the $\varepsilon_{\mathrm{t}}$ or $\varepsilon_{\mathrm{t}} \sim \mathrm{NID}\left(0, \sigma_{\mathrm{t}}^{2}\right)$. The problem of heterogeneously distributed processes is relevant since platinum prices have been found to display heteroscedasticity. In the third random walk model, the error terms are uncorrelated and neither independent nor identically distributed as mentioned in research of Jefferis and Smith (2005). This paper will also focus on the third model, with volatilities changing over time.

Equation (1) has constant parameters and the error terms are assumed to follow the usual classical assumptions. With financial markets, the assumption of constant variance may be inappropriate as empirical evidence frequently finds that returns have a variance which changes systematically. Equation (1) cannot readily capture gradual deviations towards/ from the random walk over successive observations.

\subsection{Garch approach with time varying parameters}

Emerson et al. (1997) and Zalewska-Mitura and Hall (1999) have developed, using a GARCH approach, a test with timevarying parameters which detects changes towards/from the random walk where the error process does not have a full set of Normally independent and identically distributed (NIID) properties. The model checks for changes towards/from the random walk and allows the error process to deviate from the property of being normally independent and identically distributed. The test has three characteristics:

i. $\quad$ it checks for the random walk

ii. it detects changes from/towards the random walk

iii. it will operate with a stochastic series for which the error process might not have a full set of NIID properties. The test is based on the following set of equations to constitute the model

3. $r_{t}=\beta_{0 t}+\beta_{1 t} r_{t-1}+\delta \sigma_{t}^{2}+\varepsilon_{t}$

4. $\mu_{\mathrm{t}} \mid \psi_{\mathrm{t}-1} \sim \mathrm{N}\left(0, \sigma_{\mathrm{t}}^{2}\right)$

5. $\sigma_{\mathrm{t}}^{2}=\alpha_{0}+\alpha_{1} \varepsilon_{\mathrm{t}-1}^{2}+\gamma_{1} \sigma_{\mathrm{t}-1}^{2}$

6. $\beta_{0 t}=\beta_{0 t-1}+v_{0 t} ; v_{0 t} \sim N\left(0, \sigma_{\beta_{0}}^{2}\right)$

7. $\beta_{1 \mathrm{t}}=\beta_{1 \mathrm{t}-1}+\mathrm{v}_{1 \mathrm{t}} ; \mathrm{v}_{1 \mathrm{t}} \sim \mathrm{N}\left(0, \sigma_{\beta_{1}}^{2}\right)$

in which $\sigma_{t}^{2}$ is the conditional variance of the error term $\varepsilon_{t}$, a GARCH $(1,1)$ model. The information set available at time $t$ is denoted by $\psi_{\mathrm{t}} ; \alpha_{0}, \alpha_{1}$ and $\gamma_{1}$ are parameters needed to model the changing volatility. This model has three important characteristics. First, the intercept, $\beta_{0 \mathrm{t}}$ and slope coefficient $\beta_{1 \mathrm{t}}$ can change through time. However, the special cases where either or both of these are constant are also included. Secondly, this model incorporates an error process in which the variance changes systematically over time. Thirdly, the mean of the log return depends on its conditional variance (level of risk). The basic insight is that risk-averse investors will require compensation for holding a risky asset such as platinum. A maximum likelihood search procedure with a standard Kalman filter is used to estimate the model with equation (3), the measurement equation, and the set of equations given by (5), (6) and (7), the state equations. The Kalman filter sequentially updates coefficient estimates and generates the set of $\beta_{\mathrm{it}}{ }^{\prime} \mathrm{s}, \mathrm{i}=0,1$ and $\mathrm{t}=1 \ldots \mathrm{T}$ and their standard errors. The model recursively estimates the beta series from an initial set of priors. If the platinum log returns follows a random walk with no drift, then a $100(1-\alpha) \%$ confidence band for each of $\beta_{0 t}$ and $\beta_{1 \mathrm{t}}$ should contain zero. The method will be applied to platinum prices in this paper. The focus of this study is to find out if platinum price follow a random walk process or is mean reverting.

$\varepsilon_{\mathrm{t}}$ and $\mathrm{v}_{\mathrm{it}}, \mathrm{i}=0,1$ represent disturbances and state variables, respectively. This is somewhat unusual. The Kalman filter is being used in the context of a model with GARCH errors. The Kalman filter in its present form is not operable. This is because past values of error terms are unobservable. Nevertheless we may proceed on the basis that the model can be treated as though it were conditionally Gaussian, and we will refer to the Kalman filter as being quasioptima I (Harvey et al, 1992 cited in Moonis and Shar, ,2002). 


\subsection{Extending the model}

Zalewska-Mitura and Hall (1999) extended the model in the previous section.

The test is based on the following set of equations:

1. $\mathrm{r}_{\mathrm{t}}=\beta_{\mathrm{ot}}+\sum_{\mathrm{i}=1}^{\mathrm{p}} \beta_{\mathrm{it}} \mathrm{r}_{\mathrm{t}-\mathrm{i}}+\delta \sigma_{\mathrm{t}}^{2}+\varepsilon_{\mathrm{t}}$

2. $\varepsilon_{\mathrm{t}} \mid \psi_{\mathrm{t}-1} \sim \mathrm{N}\left(0, \sigma_{\mathrm{t}}^{2}\right)$

3. $\sigma_{\mathrm{t}}^{2}=\alpha_{0}+\alpha_{1} \varepsilon_{\mathrm{t}-1}^{2}+\gamma_{1} \sigma_{\mathrm{t}-1}^{2}$

4. $\beta_{\text {it }}=\beta_{\text {it }-1}+v_{\text {it }} ; v_{\text {it }} \sim N\left(0, \sigma_{\beta_{\mathrm{i}}}^{2}\right)$

Such a model can again be modeled using the standard Kalman filter. The parameters required to estimate timepaths of $\beta_{\text {it }}$, for $i=1,2, \ldots . ., p$ and $\delta, \alpha_{0}, \alpha_{1}, \gamma_{1}$ and all $p$ values can be found by maximizing the likelihood function. If the series $r_{t}$ is a random walk, the $100(1-\propto) \%$ confidence bands for each of the $\beta_{i t}$ 's must contain zero.

\subsection{Reasons for modeling GARCH effects}

Like many econometric time series, platinum prices exhibits periods of unusually large volatility followed by periods of relative tranquility. In such instances, the assumption of a constant variance (homoskedasticity) is inappropriate. The volatility of platinum prices displays heteroskedasticity. Modelling such varying variances involves GARCH modeling. A distinguishing feature of a GARCH model is that the error variance may be correlated over time because of volatility clustering. Thus, it is appropriate to use the GARCH model which incorporates an error process in which the variance is allowed to change systematically over time. Hence, the model can detect gradual departures from the random walk (weak form efficiency) through time.

\subsection{Building AR $(p)$ models}

An important step in the model identification process is to find the order of the auto-regressive process for the log returns. There are three basic steps to follow to fit AR (p) models to time series data. These steps involve plotting the data, possibly transforming the data, identifying the dependence orders of the model, parameter estimation, and diagnosis and model choice. The Box Jenkins methodology using auto-correlations is used to identify the order of the model Tsay (2002).

\subsection{Model selection for ADF Tests}

The lag order, in addition to a sample size can affect the finite sample behavior of the ADF test. Proper correction for the lag effect in implementing the ADF test is desirable. Because appropriate values for the ADF test can be easily computed with desirable accuracy from response surface equation for any sample size and lag length, the analysis should be useful in practical applications (Cheung and Lai, 1995). The number of the augmenting lags $(p)$ is determined by minimizing the Schwartz Bayesian information Criterion (SBI) or minimizing the Alkaike Information Criterion (AIC). In this study the SBI is used and the software automatically selects the appropriate lag length and hence the model.

\section{Empirical Results}

This section discusses data source and data analysis. The data consist of platinum prices. The section also discusses the results of the ADF tests to the random walk process. Lastly, results from the GARCH model with time-varying parameters approach are also discussed and compared to the ADF tests.

\subsection{Data}

The data used in this study is monthly platinum prices from January 1970 to April 2012 with a total of 509 observations and is quoted in US dollars. The data is a monthly platinum price per ounce and is available on the website www.wikispot.org. The data series is also transformed into monthly log returns series by taking the first difference of the logarithm of the prices to give the log returns.

Descriptive statistics for the monthly platinum prices and returns are shown in Table 1. The value of the kurtosis for returns is very high and greater than three. This indicates that the distribution is leptokurtic, that is, it is fat tailed. This 
shows that the returns display financial characteristics of volatility clustering and leptokurtosis. The skewness for both prices and returns is positive indicating that the distribution has a long right tail. The high values of kurtosis for the returns suggest that extreme price changes occurred frequently during the sampling period.

Table 1: Descriptive statistics for the monthly platinum prices and returns

\begin{tabular}{|c|c|c|}
\hline & Prices & Returns \\
\hline Mean & 569.98 & 0.001909 \\
\hline Median & 416.90 & 0.001969 \\
\hline Standard deviation & 438.30 & .034821 \\
\hline Minimum & 97.5 & -0.215647 \\
\hline Maximum & 2180.7 & 0.01503 \\
\hline Skewness & 1.5448 & -0.0286 \\
\hline Kurtosis & 4.67889 & 8.341578 \\
\hline Jacque-Bera & $264.8097(0.0000)$ & $610.8149(0.0000)$ \\
\hline
\end{tabular}

Graphical plots of platinum prices and returns are shown in Figure 1. The graphical plot shows that the monthly platinum prices are not stationary while the plot for the returns shows that volatility occurs in bursts which indicate volatility clustering.
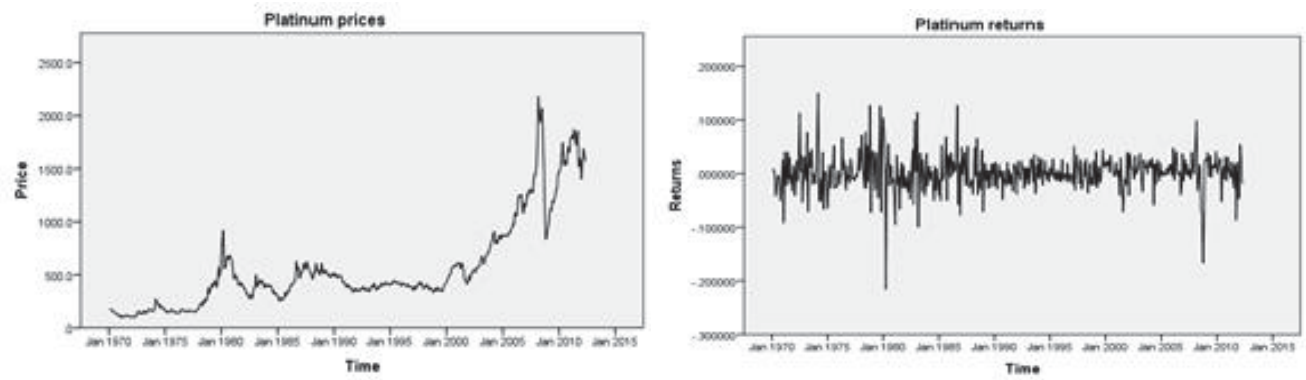

Figure 1: Plot of monthly platinum prices and log returns

\subsection{The ADF test for the data in the period 1970 to 2012}

The ADF test is used to test for stationarity in the data set from 1970 to 2012. Conclusions are made in line with Geman's (2007) paper. The ADF test statistic for untransformed platinum price is -2.832881 with $p$ value of 0.1861 . At $5 \%$ significance level, the null hypothesis of non-stationary (unit root) is not rejected implying that platinum prices are nonstationary. Non stationarity implies the random walk (Geman, 2007).

Similar results are obtained for log platinum price data from January 1970 to May 2012. The ADF test statistic is 2.570121 with $p$ value 0.2944 . The $p$ value is greater than $5 \%$, the null hypothesis of unit root is not rejected. This result implies that the log platinum price is non- stationary and is thus a random walk.

\subsection{Results from the GARCH model with time varying parameters}

The results of using the GARCH model with time varying parameter are presented in this section. Figures 2, 3 and 4 present the results of the changes towards / from the random walk. The figures show the paths of the estimated $\beta_{\mathrm{it}}{ }^{\prime} \mathrm{s}, \mathrm{i}=0,1,2$ coefficient (see equation (8)) with their respective 95 per cent confidence bands.

For the period 1970 to 2012, the best model using the Box Jenkins methodology is an AR (2) model:

$r_{t}=\beta_{0 t}+\beta_{1 t} r_{t-1}+\beta_{2 t} r_{t-2}+\mu_{t}$ 


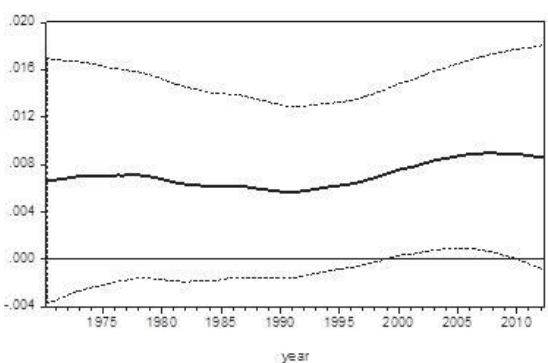

Figure 2. $\widehat{\beta}_{0 t}$, Drift parameter for platinum from 1970 to 2012. The estimates of $\widehat{\beta}_{0 t}$ are shown by a solid bold line and its confidence limits by dotted lines.

Consider Figure 2, which represents the results of the estimated drift parameter $\widehat{\beta}_{0 \mathrm{t}}$ for the period 1970 to 2012 . The estimate $\widehat{\beta}_{0 \mathrm{t}}$, has an initial value of 0.0067 and is not significantly different from zero considering its 95 percent confidence limit, except between April 1999 and January 2010. The parameter remains insignificant for the rest of the period and has a value of 0.0087 in May 2012. There is a positive drift during the period between April 1999 and January 2012.

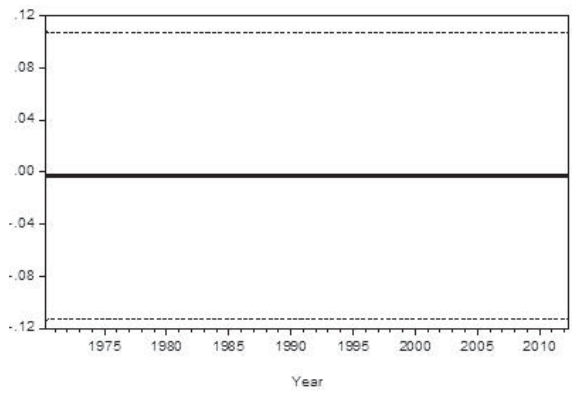

Figure 3. $\widehat{\beta}_{1 \mathrm{t}}$ estimates for platinum price from 1970 to 2012. The estimates of $\widehat{\beta}_{1 \mathrm{t}}$ are shown by a solid bold line and its confidence limits by dotted lines

Figure 3 shows the results for the parameter $\widehat{\beta}_{1 \mathrm{t}}$ for the period 1970 to 2012. The estimate $\widehat{\beta}_{1 \mathrm{t}}$ has a constant value of 0.0003 and is insignificantly different from zero at 0.05 significance level from considering its 95 percent confidence limits.

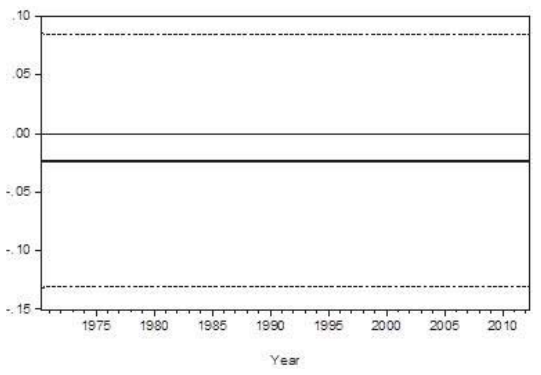

Figure 4. $\widehat{\beta}_{2 t}$ for platinum price 1970 to 2012 . The estimates of $\widehat{\beta}_{2 t}$ are shown by a solid bold line and its confidence limits by dotted lines.

Figure 4 shows the results for the parameter $\widehat{\beta}_{2 t}$ for the period 1970 to 2012 . The estimate $\widehat{\beta}_{2 t}$ has a constant value of 0.023 and is insignificantly different from zero at 0.05 level. Platinum returns follow a random walk from January 1970 to May 2012. The platinum market is weak form efficient and shows no tendency to move away from the random walk.

This results shows that platinum returns follow a random walk during the period under consideration. The results 
are in line with the results by Jeffries and Smith (2005) on the South African stock market which is weak form efficient market ( $a$ random walk). It should be noted that South Africa is one of the largest producers of platinum in the world.

\section{Conclusion}

In this study, an attempt has been made to determine whether platinum price is mean reverting or is a random walk process. Two approaches namely, the Augmented Dickey-Fuller (ADF) test and the GARCH model with time-varying properties are used. Before carrying out formal Augmented Dickey-Fuller (ADF) tests, the autocorrelation function (ACF) correlogram of platinum price and log platinum price are examined to investigate stationarity.

This paper uses current monthly data on platinum prices up to the month of May 2012. Most researchers investigate random walk behavior and market efficiency in commodities markets by mainly using the variance tests, unit root tests like the Augmented Dickey-Fuller (ADF), Philips-Peron (PP) ,Kwiatkowski, Philips, Schmidt and Shin (KPSS) and LM test and the multiple variance ratio test (MVR). The GARCH model with time-varying parameters is an interesting alternative. It also caters for volatility clustering which is more pronounced in commodity and stock prices. The GARCH model with time-varying parameters approach shows the presence of random walk in log platinum prices over the period January 1970 to May 2010. The drift parameter is significant over the period April 1999 to January 2010. The results obtained in this paper are are in line with the results by Jeffries and Smith (2005) of the South African stock market which is shown to be weak form efficient (random walk). It should be noted that South Africa is one of the largest producers of platinum in the world.

SPSS, $R$ and EViews were used in this paper to produce figures and results of various tests.

\section{References}

Andersson, H. (2007). Are commodity prices mean reverting? Applied Financial Economics, 17, 769-783.

Bessembinder, H., Coughenour, J., Seguin, P. \& Smoller, M. (1995). Mean reversion in equilibrium asset prices: Evidence from the futures term structure. Journal of Finance, 50, 361-375.

Campell, J.Y., Lo, A.W. \& Mackinaly, A.C. (1997). The Econometrics of Financial Markets, Princeton, Princeton Univerity Press.

Chen, Y.C., Rogoff, K. \& Rossi, B. (2008). Can exchange rates forecast commodity prices? Working Paper.

Emerson, R., Hall, .SG.and Zalaweska-Mitura, A. (1997).Evolving Market Efficiency with an application to some Bulgaria shares. Economics of planning, 30(1), 75-90.

Geman, H. (2007). Mean Reversion versus Random Walk in Oil and Natural Gas Prices. United Kingdom: University of London.

Hiller, D., Draper, P. \& Faff R (2006). Do Precious Metals Shine? An Investment

Perspective. Financial Analysts Journal. 62: 98-106.

Jefferies, K. \& Smith, G. (2005). The changing efficiency of African stock markets. South African Journal of Economics, 73, 54-67.

Lo, A.W. \& Mackinaly, A.C. (1988).Stock Markets Prices Do Not Follow Random Walks: Evidence from a Simple Specification Test. Review of Financial Studies, 1(1), 41-66.

Moonis, S.A. \& Shar, A. (2002). Testing for time variation in beta in India. Journal of Emerging Markets Finance. 2(2): 163-180.

Nwala, K. (2011). Do commodity markets follow a random walk? An application of the LM unit root tests. Working paper, Elizabeth City State University.

Pindyck, R.S. (1999). The long run evolution of energy prices. The Energy Journal, 20:1-27.

Taylor, N. (1998). Precious metals and inflation. Applied Financial Economics. 8: 201-210.

Tsay, RS. (2002). Analysis of Financial Time Series. New York: Wiley

Zalewska-Mitura, A. \& Hall, S.G. (1999). Examining the first stages of market performance: a test for evolving market efficiency. Economics Letters. 64:1-12.

The website: www.wikiposit http://wikiposit.org/w?filter=Finance/Futures/Metals/Platinum/ [accessed on 24July 2012]

Tsay, R. S. (2010). Analysis of Financial Time Series, Third Edition. Wiley \& Sons. 